\title{
FLOATING SOLAR PANEL ALONG WITH SUN TRACING SYSTEM-A REVIEW
}

\author{
Mr. Kushal Sardar, Mr. Prashant Singh, Mr. Shubham Sawant, Mr. Devang Soni \\ Department of Computer Engineering, Xavier Institute of Engineering, Mumbai University, India \\ tataisardar2000@gmail.com, prashantsingh93840@gmail.com,sh92963@gmail.com,devangsoni2310@gmail.com
}

\begin{abstract}
With the increasing demands of fossil fuels and energy sources, everyone is looking for alternative sources of energy. With the use of solar energy this hunger for power can be reduced to an extent, but it is not being utilized to its maximum extent. Normal solar panels are around 15\% - 22\% efficient and majority of times it is not that efficient. Floating solar panel can be used in place of conventional solar panel and the efficiency can be maximized. However using this solar system have some of issues like installation which is a big issue, cost factor, fragile as well as care should be taken while windy environment. Beside these drawbacks it has its many advantages as well. Floating solar panels can be used in vast area and can be beneficial for the industries too. Now a days the human race depends on energy to live their life. As we know, we live in a country of 1.3 billion People and as we move more towards the modernization more energy or electricity is needed. We cannot depend on fossil fuel and coal as there are only limited amount of fossil Fuel present on earth and so we have to turn towards renewable source of energy i.e. Solar, wind, hydroelectricity.

As we know our country is headed Towards production of solar energy by building different solar power plants like, Bhadla solar park -2,250mw (Rajasthan)(which is the largest solar power plant in world ), Shakti Sthala solar power project 2,050mw(Karnataka), Ultra mega solar park -1000mw (Andhra Pradesh) Rewa solar power project -750mw (Madhya pradesh), Kamuthi solar power plant -648mw (Tamil Nadu). But as we see there is a problem with the Technology, that is it takes more space which is not available in city and that's why we have come with this idea of our project.
\end{abstract}

\section{INTRODUTION}

Fuels are getting consumed at a rapid rate and the total fossil fuels which mankind have are limited and will be extinct in the coming years. Every country is looking for alternatives to shift in order to reduce the consumption of fossil fuels. Solar panel came into the pattern as solar energy is available in abundance and can be harnessed at a very cheap rate as compared to other forms of fuels. The conventional solar panels require huge amount of land and that too inclined at a specific angle. So, this requires some manpower and resources to clear the ground at certain elevation. Whereas floating solar panels does not require such type of huge land requirements and inclination of the land.
As we know there is a scarcity of land in major urban cities like Mumbai, Chennai, Bangalore, Delhi and fossil fuels are also coming to extinct and it is also seen that, solar energy generated in city are less because of scarcity of land and location, the only place where sunlight are commonly seen are on the terrace of building.

Since we cannot place hundreds of panels on terrace buildings, as a solution to this issue our project comes to scene.

People started creating floating solar Panels in India under various corporations like Blue India company ltd, National thermal power corporation (NTCP). Now one may ask why are making this project?, as it is already been used on large scale? 
The answer is simple because there is a flaw in that system that is, they are set at one position to collect solar energy but the sun is constantly moving and due to which efficiency of this solar panels is less. As we see the solar panel industry, it has really progressed in a fast pace. They have created two solar panels i.e. Fixed solar panels and rotating solar panels.

Fixed solar panels are set to one position and left there. Whereas rotating solar panels are of two types

1.Single axis rotating solar panels 2. Dual axis rotating solar panels

In the rotating solar panels there are two types sun tracing techniques i.e. Active tracing and passive tracing techniques.

\section{WORKING PRINCIPLE}

Resistance of LDR depends on intensity of the light and it varies according to it. The higher is the intensity of light, lower will be the LDR resistance and due to this the output voltage lowers and when the light intensity is low, higher will be the LDR resistance and thus higher output voltage is obtained.

A potential divider circuit is used to get the output voltage from the sensors (LDRs). The circuit is shown here.

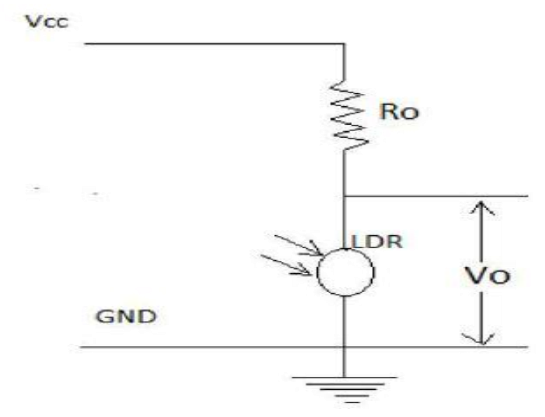

The LDR senses the analog input in voltages between 0 to 5 volts and provides a digital number at the output which generally ranges from 0 to 1023 .

Now this will give feedback to the microcontroller using the Arduino software (IDE).

The servo motor position can be controlled by this mechanism which is discussed later in the hardware model.

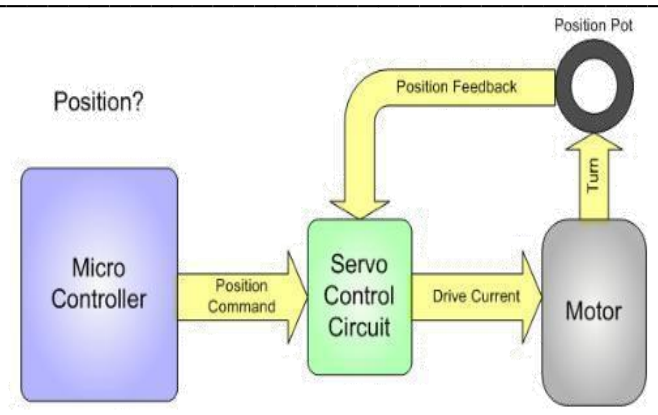

The tracker finally adjusts its position sensing the maximum intensity of light falling perpendicular to it and stays there till it notices any further change.

The sensitivity of the LDR depends on point source of light. It hardly shows any effect on diffuse lighting condition.

\section{BASIC CIRCUIT DIAGRAM}

An overview of the required circuit for the Dual-axes solar tracker is shown here. The $5 \mathrm{~V}$ supply is fed from an USB 5V dc voltage source through Arduino Board. Servo X: Rotates solar panel along $X$ direction $\longleftrightarrow$

Servo Y: Rotates solar panel along Y direction

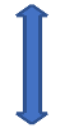

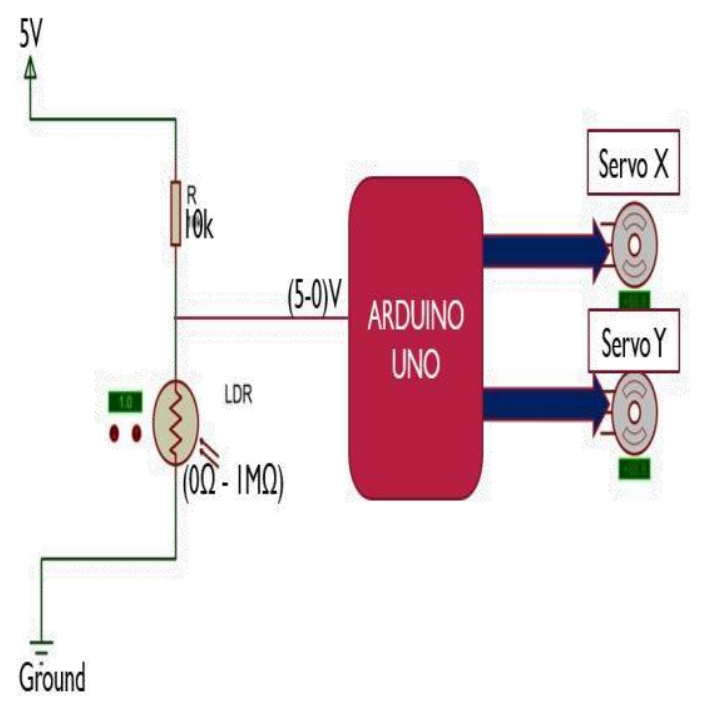




\section{FLOW CHART}

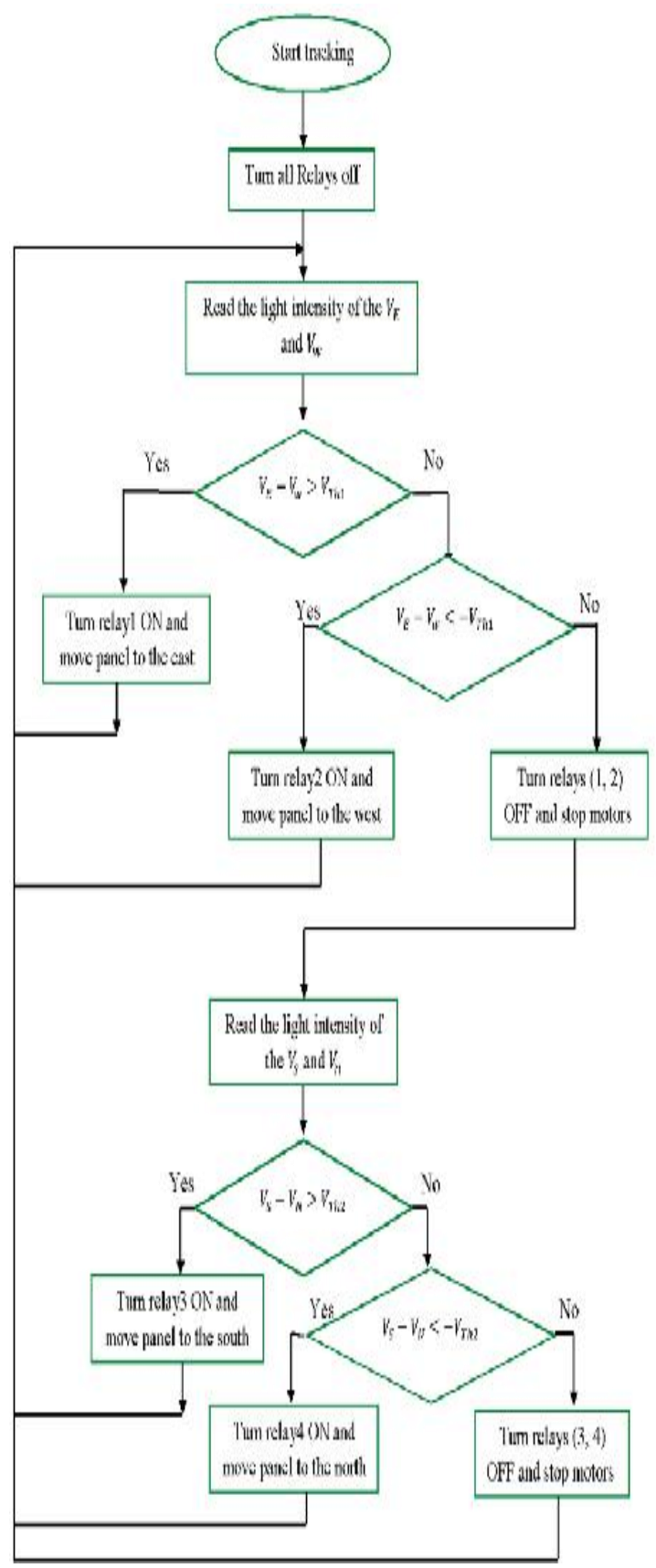

5. EXPLANATION OF THE BLOCK DIAGRAM:

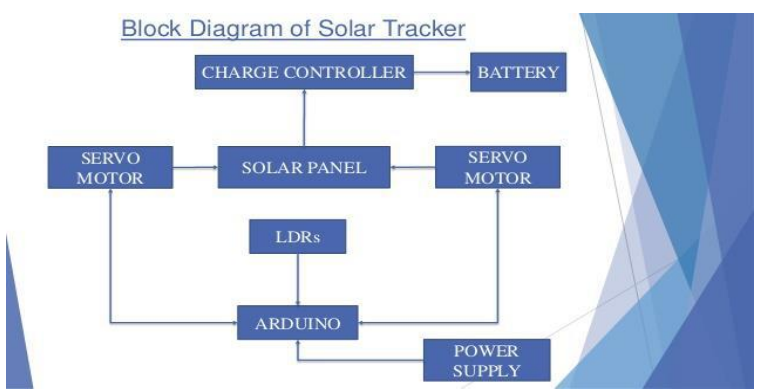

As we see in the block diagram, there are three Light Dependent Resistors (LDRs) which are placed on a common plate with solar panel. Light from a source strikes on them by different amounts. Due to their inherent property of decreasing resistance with increasing incident light intensity, i.e. photoconductivity, the value of resistances of all the LDRs is not always same.

Each LDR sends equivalent signal of their respective resistance value to the Microcontroller which is configured by required programming logic. The values are compared with each other by considering a particular LDR value as reference.

One of the two dc servo motors is mechanically attached with the driving axle of the other one so that the former will move with rotation of the axle of latter one. The axle of the former servo motor is used to drive a solar panel. These two-servo motors are arranged in such a way that the solar panel can move along $\mathrm{X}$-axis as well as $\mathrm{Y}$-axis.

The microcontroller sends appropriate signals to the servo motors based on the input signals received from the LDRs. One servo motor is used for tracking along $\mathrm{x}$-axis and the other is for $\mathrm{y}$-axis tracking.

In this way the solar tracking system is designed.

\section{ARDUINO UNO}

The Arduino Uno is a microcontroller board based on the ATmega328. Arduino is an open-source, prototyping platform and its simplicity makes it ideal for hobbyists to use as well as professionals. The Arduino Uno has 14 digital input/output pins (of which 6 can be used as PWM outputs), 6 analog inputs, a $16 \mathrm{MHz}$ crystal oscillator, a USB connection, a power jack, an ICSP header, and a reset button. It contains everything needed to support the microcontroller; simply connect it to a computer with a USB cable or power it with a AC-to-DC adapter or battery to get started.

The Arduino Uno differs from all preceding boards in that it does not use the FTDI USB-to-serial driver chip. 
Instead, it features the Atmega8U2 microcontroller chip programmed as a USB-to-serial converter.

"Uno" means one in Italian and is named to mark the upcoming release of Arduino 1.0. The Arduino Uno and version 1.0 will be the reference versions of Arduino, moving forward. The Uno is the latest in a series of USB Arduino boards, and the reference model for the Arduino platform.

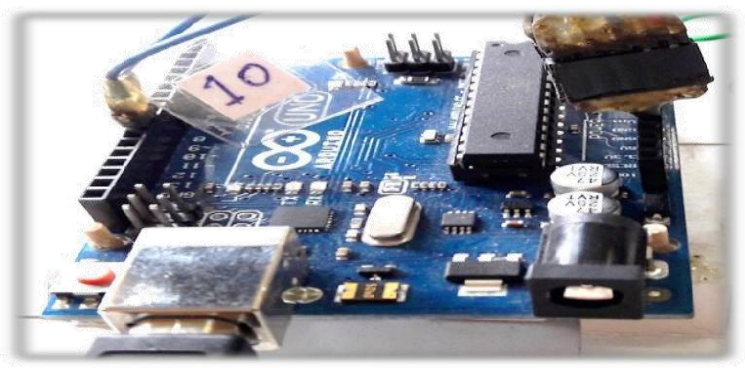

THE ATMEGA328P-PU ATMEL 8 BIT 32K AVR MICROCONTROLLER

(The Atmel®picoPower®ATmega328/P is a lowpower CMOS 8-bit microcontroller based on the AVR® enhanced RISC architecture.)

\subsection{PIN DIAGRAM}

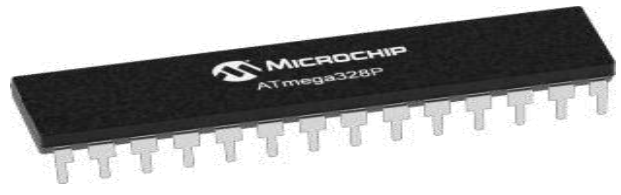

\subsection{PIN LAYOUT OF ATMEGA328P}

Pin layout of ATmega328p is showed below:

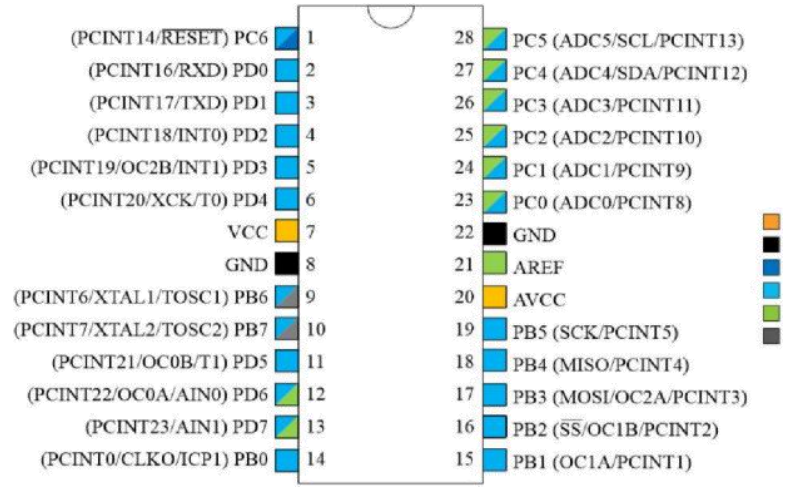

\subsection{AVR CPU CORE ARCHITECTURE}

The main function of the CPU core is to ensure correct program execution. The CPU must therefore be able to access memories, perform calculations, control peripherals, and handle interrupts. Flash, EEPROM, and SRAM are all integrated onto a single chip, removing the need for external memory in most applications. Some devices have a parallel external bus option to allow adding additional data memory or memory-mapped devices. Almost all devices (except the smallest Tiny AVR chips) have serial interfaces, which can be used to connect larger serial EEPROMs or flash chips.

\subsection{BLOCK DIAGRAM OF AVR ARCHITECTURE}

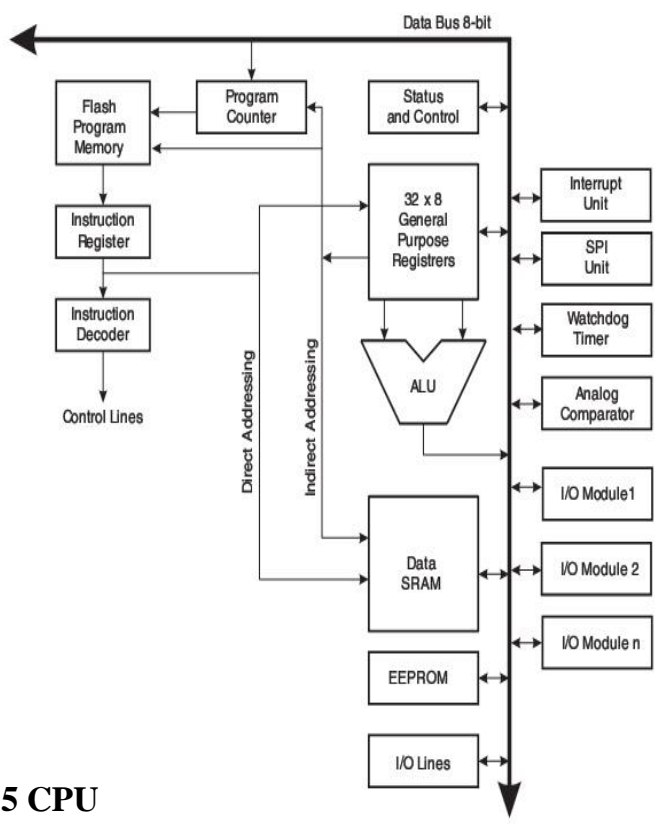

The CPU of the AVR microcontroller is same but so simple like the one in a computer. The main purpose of the CPU is to confirm correct program performance. Therefore, the CPU must be able to access perform calculations, memories, control peripherals \& handle interrupts. The CPUs of Atmel's 8-bit and 32-bit AVR are based on an innovative "Harvard architecture" thus every IC has two buses namely one instruction bus and data bus. The CPU reads executable instructions in instruction bus, wherein the data bus, is to read or write the corresponding data. The CPU core of the AVR consists of the ALU, General Purpose Registers, Program Counter, Instruction Register, Instruction Decoder, Status Register and Stack Pointer

\subsection{Flash Program memory}

The program of the AVR microcontroller is stored in non-volatile programmable Flash program memory 
which is just similar to the flash storage in your SD Card or Mp3 Player. The Flash program memory is separated into two

units. The first unit is the Application Flash section. It is where the program of the AVR is stored. The second section is named as the Boot Flash section and can be fixed to perform directly when the device is powered up. One significant fact to note is that the microcontrollers Flash program memory has a resolution of at least 10,000 writes/erase cycles.

\subsection{SRAM}

The SRAM (Static Random-Access Memory) of the AVR microcontroller is just like computer RAM. While the registers are used to execute calculations, the SRAM is used to supply data through the runtime. This volatile memory is prearranged in 8-bit registers.

\subsection{EEPROM}

The term EEPROM stands for Electrically Erasable Read-Only Memory is like a non-volatile memory, but you can't run a program from it, but it is used as longtime storage. The EEPROM doesn't get removed when the IC loses power. It's a great place for storing data like device parameters and configuration of the system at runtime so that it can continue between resets of the application processor. One significant fact to note is that the EEPROM memory of the AVR has a limited lifetime of 100,000 writes / EEPROM page - reads are limitless. Keep this in mind in your application and try to keep writing to a minimum, so that you only write the small amount of info required for your application every time you update the EEPROM.

\subsection{Digital I/O Modules}

The digital I/O modules let digital communication or logic communication with the AVR microcontroller and the exterior world. Communication signals are that of TTL/CMOS logic.

\subsection{Analog I/O Modules}

Analog I/O modules are used to input or output analog information from or to the exterior world. These modules comprise analog comparators and analog-todigital converters (ADC).

\section{Interrupt Unit}

Interrupts have enabled the microcontroller to monitor particular events in the background while performing and application program \& respond to the occurrence if required pausing the unique program. This is all synchronized by the interrupt Unit.

\section{Timer}

Most AVR microcontrollers have at least one Timer or Counter module which is used to achieve timing or counting operations in the microcontroller. These comprise time stamping, counting events, measuring intervals, etc.

\section{LDR}

- - It is a photo-resistor. It is a device whose resistivity is a function of the incident electromagnetic radiation. Hence, they are light sensitive devices. They are also called as photo conductors, photo conductive cells or simply photocells.

- $\quad$ They are made up of semiconductor materials having high resistance.

- $\quad$ LDR works on the principle of photo conductivity.

Photo conductivity is an optical phenomenon in which the material's conductivity is increased when light is absorbed by the material.

The most common type of LDR has a resistance that falls with an increase in the light intensity falling upon the device (as shown in the image above). The resistance of an LDR may typically have the following resistances:

Daylight $=5000 \Omega$

Dark $=20000000 \Omega$

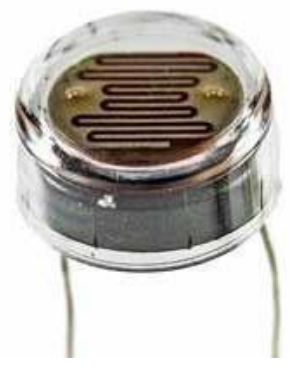

Therefore, it can be seen that there is large variation between these figures. If this variation is plotted on a graph, something similar to figure given below can be seen. 


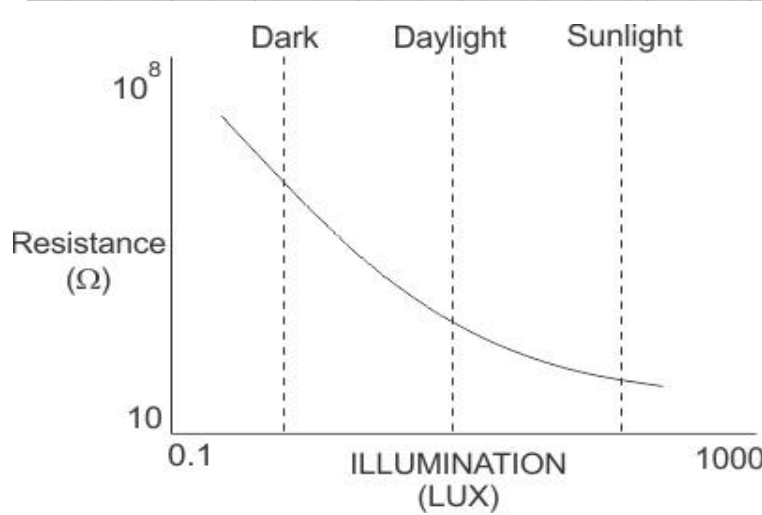

\section{SERVO MOTOR}

A DC servo motor consists of a small DC motor, feedback potentiometer, gearbox, motor drive electronic circuit and electronic feedback control loop. It is more or less similar to the normal DC motor. The stator of the motor consists of a cylindrical frame and the magnet is attached to the inside of the frame. A brush is built with an armature coil that supplies the current to the commutator. At the back of the shaft, a detector is built into the rotor in order to detect the rotation speed.

With this construction, it is simple to design a controller using simple circuitry because the torque is proportional to the amount of current flow through the armature.

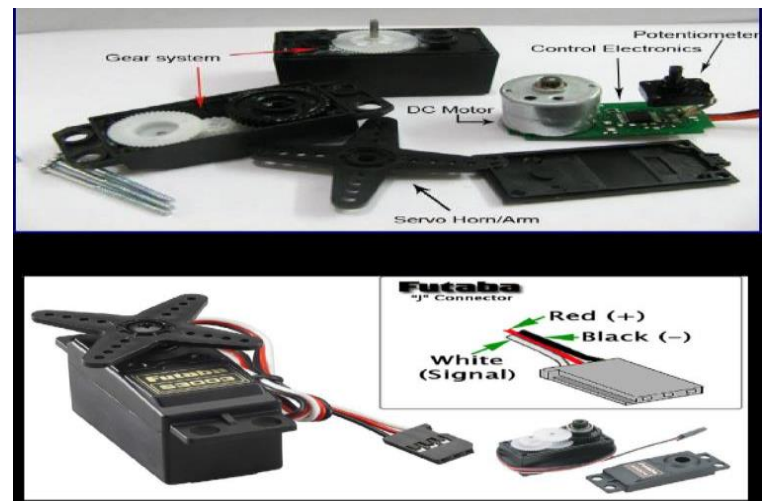

\section{ABOUT SOLAR PANEL AND CONNECTED}

\section{LOAD}

Solar panel is placed at the top and connected to a load directly. The load may a led or a voltmeter which could be connected to get the exact voltage which depends on the intensity of light falling on the panel and the position of the tracker.

Concentrated solar photovoltaics' and have optics that directly accept sunlight, so solar trackers must be angled correctly to collect energy. All concentrated solar systems have trackers because the systems do not produce energy unless directed correctly toward the sun.

The solar panel is just a mere device to accept the light radiation which is purely controlled by LDR sensors and the load connected depends upon the rating of the panel used.

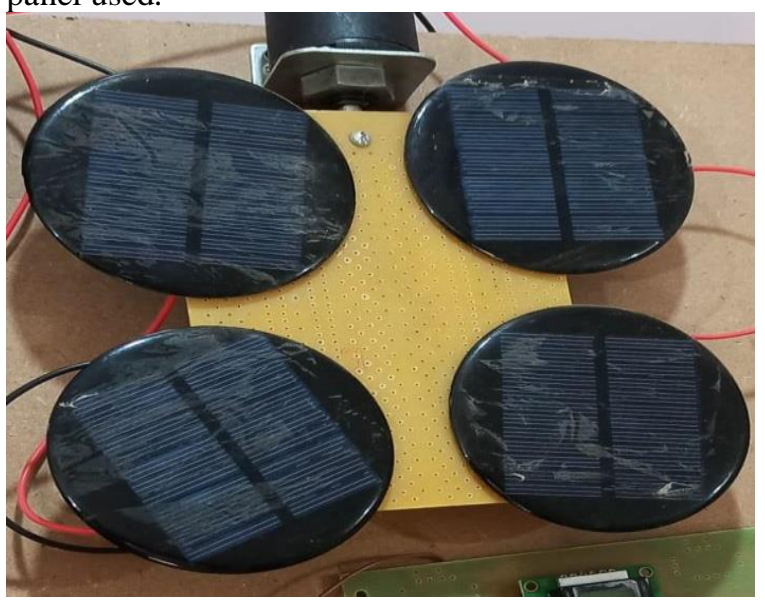

\section{RESULTS AND DISCUSSION}

In this Dual Axis Solar Tracker, when source light falls on the panel, the panel adjusts its position according to maximum intensity of light falling perpendicular to it. The objective of the project is completed. This was achieved through using light sensors that are able to detect the amount of sunlight that reaches the solar panel. The values obtained by the LDRs are compared and if there is any significant difference, there is actuation of the panel using a servo motor to the point where it is almost perpendicular to the rays of the sun. This was achieved using a system with three stages or subsystems. Each stage has its own role. The stages were;

An input stage that was responsible for converting incident light to a voltage.

A control stage that was responsible for controlling actuation and decision making.

A driver stage with the servo motor. It was responsible for actual movement of the panel. 
The input stage is designed with a voltage divider circuit so that it gives desired range of illumination for bright illumination conditions or when there is dim lighting. The potentiometer was adjusted to cater for such changes. The LDRs were found to be most suitable for this project because their resistance varies with light. They are readily available and are cost effective. Temperature sensors for instance would be costly.

The control stage has a microcontroller that receives voltages from the LDRs and determines the action to be performed. The microcontroller is programmed to ensure it sends a signal to the servo motor that moves in accordance with the generated error.

The final stage was the driving circuitry that consisted mainly of the servo motor. The servo motor had enough torque to drive the panel. Servo motors are noise free and are affordable, making them the best choice for the project.

\section{CONCLUSION}

In this 21 st century, as we build up our technology, population \& growth, the energy consumption per capita increases exponentially, our energy resources (e.g. fossils fuels) decrease rapidly. So, for sustainable development, we have to think alternative methods (utilization of renewable energy sources) in order to fulfil our energy demand.

In this project, Solar Panel with Dual Axis Solar Tracker, we've developed a demo model of solar tracker to track the maximum intensity point of light source so that the voltage given at that point by the solar panel is maximum. After a lot of trial and errors we've successfully completed our project and we are proud to invest some effort for our society. Now, like every other experiment, this project has couple of imperfections.

- Our panel senses the light in a sensing zone, beyond which it fails to respond.

- If multiple sources of light (i.e. diffused light source) appear on panel, it calculates the vector sum of light sources \& moves the panel in that point.

* The efficiency of the panels has increased dramatically and thus will be overall beneficial to us.

- Natural evaporation helps to keep the temperature low to increase the efficiency also.

- Dust accumulation does not take place which is major factor for the solar panels to work.
This project was implemented with minimal resources. The circuitry was kept simple, understandable and user friendly.

\section{AVENUES FOR FURTHER WORK}

With the available time and resources, the objective of the project was met. The project is able to be implemented on a much larger scale. For future projects, one may consider the use of more efficient sensors, which should also be cost effective and consume little power. This would further enhance efficiency while reducing costs. If there is the possibility of further reducing the cost of this project, it would help a great deal. This is because whether or not such projects are embraced is dependent on how cheap they can be. Shading has adverse effects on the operation of solar panels. Shading of a single cell will have an effect on the entire panel because the cells are usually connected in series. With shading therefore, the tracking system will not be able to improve efficiency as is required.

\section{REFERENCES}

[1] Solar Tracking Hardware and Software by Gerro $J$ Prinsloo

[2] Design and Implementation of a Sun Tracker with a Dual-Axis Single Motor

[3] Sensors and Transducers, Second

Edition..."'D.Patranabis"

[4] Atmel ATmega48A/PA/88A/PA/168A/PA/328/Pdatasheet

[5] Utilisation of Electrical Power. Author, Er. R. K. Rajput.

[6] Arduino Programming Book. Author, Brian W. Evans 